\title{
Efficacy and patient tolerability of travoprost BAK-free solution in patients with open-angle glaucoma and ocular hypertension
}

This article was published in the following Dove Press journal:

Clinical Ophthalmology

27 July 2010

Number of times this article has been viewed

\author{
Sophia K Mirza \\ Sandra M Johnson \\ Department of Ophthalmology, \\ University of Virginia, Charlottesville, \\ Virginia, USA
}

\begin{abstract}
The medical treatment of glaucoma has evolved significantly over the past several decades. The main driving forces behind this evolution are the safety profiles and efficacy of these medications. Prostaglandin (PG) analogues are shown to be superior to older drugs in both efficacy and tolerability. Though there are much fewer side effects that manifest after using PG analogues, the adherence and compliance to medication regimens are surprisingly lower than expected. A commonly sited reason is the ocular irritation and inflammation with these medications. Much of this inflammation can be attributed to the preservative, benzalkonium chloride (BAK). The chronic clinical and subclinical inflammation becomes increasingly detrimental when filtration surgery fails from bleb fibrosis secondary to this hypercellularity. A BAK-free formulation of a PG analogues recently became available. BAK-free travoprost is reviewed here. It has demonstrated equal efficacy and less ocular surface toxicity than its preserved counterparts It is expected to serve as an instrumental resource in managing ocular hypertension and glaucoma in patients who demonstrate significant sensitivity to BAK. More randomized, controlled, double-blind studies are encouraged to evaluate its improved safety and tolerability.
\end{abstract}

Keywords: glaucoma, benzalkonium chloride, Travatan Z, sofZia

\section{Introduction}

This is a literature review, which discusses medical therapy of glaucoma and focuses on adverse effects of the topical drugs. An overview of the prevalence of glaucoma, antiglaucoma medications, their side effects, and consequences of these side effects is provided, with particular emphasis on prostaglandin $(\mathrm{PG})$ analogues. Preservative-induced ocular irritation and the interaction of PGs with benzalkonium chloride (BAK) are addressed. The subsequent development of preservative-free glaucoma medications and their efficacy and safety profile are then examined. Newly available travoprost preserved with sofZia ${ }^{\mathrm{TM}}$ (Alcon Laboratories, Inc, Fort Worth, Texas, USA) is described, and the research evaluating the efficacy and safety profile of this drug is reviewed.

\section{Epidemiology}

Worldwide, approximately 60.5 million people have glaucoma, based on a literature search of over 2,000 studies. Open-angle glaucoma and angle-closure glaucoma were measured by optic disc changes, and visual field test abnormalities did not account for intraocular pressure (IOP); 79.6 million people are expected to have glaucoma by 2020. Glaucoma is a leading cause of blindness, second only to cataracts. Data indicate that there is a propensity for Asians and females to develop glaucoma. ${ }^{1}$ There is a large body of literature that analyzes prevalence data globally, and despite 
variations in extrapolating numbers from studies and testing measures, there is no doubt glaucoma is an increasingly common pathology. ${ }^{2,3}$ Recent reports from the World Health Organization indicate that of the 37 million people who are currently blind, 4.5-5 million are blind due to glaucoma. ${ }^{4}$ Prevalence rates also increase as the population grows older. Of persons older than 40 years, $2.4 \%$ have glaucoma, and this increases to $7 \%$ among those older than $70 .{ }^{5}$ In the United States alone, the prevalence of glaucoma in individuals 40 years or older is 2.2 million and is estimated to increase to 3.6 million by 2020 due to the rapidly aging population. There is a significant difference in prevalence rates based on race; African American individuals are 3 times more likely to have glaucoma than Caucasian individuals. ${ }^{6}$ Among individuals of Caucasian decent who are 73 and 74 years old, the prevalence of glaucoma was shown to be $3.4 \%$; the rate increased to $9.5 \%$ among Caucasians who were older than 73 or 74 years. Among participants of African American ethnicity, rates were $5.7 \%$ for those who were 73 and 74 and increased to $23.2 \%$ among those older than $75 .^{7}$ It is also estimated that this disease process costs the US health care system $\$ 2.5$ billion, and individuals undergoing treatment spend between US \$600 and US \$2,500 per person annually, indicating a significant burden on the population. ${ }^{8}$ Based on the copious epidemiologic data available, it is expected that glaucoma will be among the leading diseases treated by ophthalmologists worldwide.

\section{Use of medications for glaucoma}

Among clinicians, it is well understood that medical therapy for open-angle glaucoma is most often the initial therapy for newly diagnosed patients and will continue to be used, often for decades, as long-term management. The Ocular Hypertension Treatment study demonstrated the efficacy of medications as they significantly reduced the rate of developing glaucoma among those with elevated IOP; $9.5 \%$ of untreated participants and only $4 \%$ of treated subjects developed glaucoma. This risk factor is especially pronounced among African Americans who participated in the trial. ${ }^{9}$ Reducing IOPs is shown to be imperative to slow disease progression for those with open-angle glaucoma, normaltension glaucoma, or just ocular hypertension. ${ }^{10}$ For several reasons, including easier accessibility compared with surgery and often similar outcomes regarding IOP of surgery and medication, medications remain the first line of therapy. ${ }^{11}$ Among these medications, PG analogues exhibit several advantages over other medical therapies. This class of drugs is able to decrease IOPs by up to $33 \%$, which is a greater decrease than other classes reviewed, including $\beta$-blockers, $\alpha 2$ adrenergics, and carbonic anhydrase inhibitors. ${ }^{12}$ There is a higher rate of adherence to treatment regimens as these drugs require only once-a-day dosing. The pressure-lowering effects can last up to 2 days and have a short half-life, which reduces the systemic side effect profile. The mechanism of action for the 3 major PG analogues or prostamides available is an increase in both trabecular and uveoscleral outflow, as they may work through a pressure-sensitive and/or pressureinsensitive pathway. ${ }^{13}$ Two of the PG analogues are prepared as prodrug isopropyl esters, which assist in accessing the cornea so that they can undergo hydrolyzation by esterase, which in turn allows them to enter the aqueous humor. There is minimal to no difference in the pressure-lowering effects of latanoprost and travoprost long term, and travoprost may maintain lower pressures in the late afternoon as compared with latanoprost. Ocular hyperemia was associated more with travoprost, though this was not significant. ${ }^{14}$ As the importance of medical therapy is quite established in clinical practice and medications are shown to be efficacious, the concern of medication safety remains uncertain. Dry eye is more prevalent among those with glaucoma than in the general population. ${ }^{15}$

\section{Dry eye in the general population}

Population-based studies, which look at the pervasiveness of dry eye disease (DED), found that DED is a common problem among several sectors of the population including postmenopausal women and the elderly. Several studies conducted in the United States indicate that the prevalence of dry eye among individuals of an approximate age of 50 or older is from $7 \%$ to $33 \% .^{16-18}$ The prevalence of dry eye and dry mouth (sicca syndrome) was reported to be $27 \%$ among the elderly. In one study of 2,481 individuals between the ages of 65 and 84, there was a positive and dose-response association between dry eye or dry mouth and the use of certain medications, which could cause ocular irritation through several different mechanisms. To what extent could the signs and symptoms of dry eye be attributed to medications may not be entirely clear; however, a significant percentage of the population $(62 \%)$ had dry eye or dry mouth symptoms associated with medication usage. ${ }^{19}$ The international prevalence of dry eye syndrome, including studies conducted in Canada, Taiwan, Indonesia, Australia, and Japan, is similar to rates seen in the United States. ${ }^{18,20-23}$ The reduction of quality of life and mounting costs of DED is quite significant. One study used utility assessment scores to compare the quality of life of those with DED and other 
common pathologies. The utility scores for dry eye correlated remarkably with scores for several systemic pathologies. Mild dry eye had utility scores comparable with psoriasis, scores for moderate dry eye were comparable with those for moderate angina, whereas severe dry eye scoring was comparable with utility scores for hip fractures. ${ }^{24}$ The costs of this debilitating condition are reflected in the large number of individuals who spend each year on lubricating solutions. In a 6-country study, the prevalence of individuals who see their ophthalmologist for dry eye was approximated at $0.1 \%$, and the yearly costs of 1,000 patients who were being treated by their ophthalmologists ranged from US\$0.27 million in France to US\$1.10 million in the United Kingdom. ${ }^{25}$ These expenses only reflect the amount being spent by individuals who have their ophthalmologist manage their symptoms; they do not reflect the likely million more people who suffer from dry eye and spend money self medicating.

\section{Dry eye associated with glaucoma medications}

Clinicians who manage glaucoma are well aware of the associated ocular surface disease (OSD) and inflammation associated with glaucoma medication. The prevalence of dry eye among these individuals is delineated in several surveys and cross-sectional studies. Although there are several studies, the diagnosis of DED is highly variable due to the flexible definitions of dry eye. ${ }^{26}$ One analysis used the Medical Expenditure Panel Survey, a national health survey, and examined the rates of dry eye, identified by diagnosis or the use of prescribed medications for dry eye. The rate of dry eye syndrome was compared between those with glaucoma, identified by the use of glaucoma medication, and nonglaucoma controls. Patients with dry eye were more commonly females and had higher rates of diabetes and hypertension. Respondents with glaucoma demonstrated dry eye $16.5 \%$ of the time, whereas nonglaucoma controls were associated with dry eye only $5.6 \%$ of the time. Importantly, among the glaucoma patients, those using adjunctive therapy reported higher rates of dry eye than those who did not; however, these data are not significant, possibly due to a lower power in the study.

PG analogues were the most commonly prescribed medication for those with and without dry eye. There was a higher ratio of people who had both dry eye and glaucoma (21\%) than those who had only dry eye $(7.4 \%)$, which is compelling data that support the concern of glaucoma medication-induced DED. This is more substantial when considering this study only in those with dry eye clinically significant enough to warrant medication, leaving out all subclinical cases. ${ }^{27}$ Other studies show that the severity of dry eye among glaucoma patients increases with the number of antiglaucoma medications being used. ${ }^{28,29}$ Patients using glaucoma medications for at least 6 months or longer demonstrated a significantly larger amount of inflammatory markers on the ocular as measured via antibodies to several cytokines. In this study, there was no significant difference in the amount of cytokines found in those who used more than 1 glaucoma medication. ${ }^{30}$

\section{Costs of hyperemia induced by PG analogues}

When researchers calculated the costs of switching from 1 PG analogues, bimatoprost, latanoprost, or travoprost, to 1 of the other 2 PG analogues based on physician-recommended medication switches due to hyperemia, there was a notable difference. The costs were calculated from the amount charged for the office visit and the amount of the new prescription. Costs for hyperemia-free patients were US\$73.67, and costs for those whose medications were changed due to hyperemia were US\$140.02. Costs were lowest for patients who started the study with latanoprost, indicating their medications were switched less often; costs were highest for travoprost, which were only 50 cents higher than for bimatoprost. ${ }^{31}$ These increased costs indicate that conjunctival hyperemia, which is clinically significant enough to switch medications, is expensive for patients and time consuming for physicians.

\section{Compliance or adherence}

Besides the costs of medication-induced OSD, patientreported ocular irritation and/or inflammation is, as described, common and should be considered an even greater factor when prescribing medications for glaucoma. ${ }^{32}$ One of the most common side effects attributed to this class of drugs is hyperemia, which has been found in nearly twothirds of patients experiencing adverse effects. ${ }^{33}$ Clinicians often report that this prevalent and seemingly benign side effect can be quite deleterious. Because hyperemia, among other side effects, is not aesthetically pleasing to patients, they are often nonadherent to their dosing schedule, leading to uncontrolled pressures and further disease progression. ${ }^{34}$ Several investigations of glaucoma medication adherence were performed. One cross-sectional study completed in Australia conducted surveys inquiring patients' compliance with medication regimens. Of the participants, $45 \%$ reported nonadherence, in which $66 \%$ were unintentional nonadherence and $17 \%$ reported either intentionally missing doses 
or both. Those who forgot doses were generally younger, had less comorbidities, and tended to report not believing in medication efficacy for glaucoma. Intentionally missing doses were associated with concerns about medications. ${ }^{35}$ A reviewer searched literature from 1980 to 2004 regarding patients' adherence and duration of continuation of utilization of ocular hypotensives. Several methods of measurement of adherence and persistence were encountered; noncompliance rates of $25 \%$ or more were frequently reported. Also reported was that nonadherence was attributed to situational/environmental reasons or side effects/ complexity of regimens of medications. In addition, the reviewer reported that less than $25 \%$ of patients were persistent over 12 months. ${ }^{36}$ Other factors that cause this characteristic adherence pattern to glaucoma medications are described in a part retrospective and part prospective study, which elucidates several variables including patients' education of glaucoma, patients' beliefs in medication efficacy, payment for medications, situational or traveling, and not acknowledging side effects. In their conclusion, they found that focusing on patients' education was associated with better adherence to medication regimens. ${ }^{33}$

\section{Hyperemia attributed to preservatives}

Ocular irritation and scleral injection associated with PG analogues have been attributed to preservatives in ophthalmic solutions. Parrish et $\mathrm{al}^{37}$ showed that after over 3 weeks of treatment, subjects who reported mild hyperemia were associated with the usage of bimatoprost $69 \%$ of the time, with travoprost $58 \%$ of the time, and with latanoprost $47 \%$ of the time. Moderate hyperemia was also reported to be associated with bimatoprost $15 \%$ of the time, with travoprost $10 \%$ of the time, and with latanoprost $6 \%$ of the time. There is much subjective and objective evidence that indicates preserved medications cause significant ocular disease. ${ }^{38}$ Some investigators distributed a survey questionnaire to patients using either preserved or preservative-free glaucoma medications for a long term and found that symptoms such as foreign body sensation, stinging, burning, and tearing were associated more with those using preserved medications. Moreover, when patients were switched to preservative-free formulations, their symptoms decreased or disappeared. . $^{39,40}$

Though preservatives are associated with a spectrum of symptomatic irritation, they are necessary to maintain sterility of multiuse ophthalmic solutions, without which they are more likely to be contaminated. ${ }^{41}$ In unpreserved ophthalmic preparations, contamination usually occurs within 1-2 weeks of twice-daily usage. ${ }^{42}$ Other studies demonstrated contamination in almost $20 \%$ of unpreserved solutions less than 8 weeks old and $40 \%$ contamination in those greater than 8 weeks old. ${ }^{43}$ BAK, a common preservative, is a quaternary ammonium compound. It is used in approximately $70 \%$ of preserved ophthalmic solutions and only $10 \%$ use other preservatives. ${ }^{44}$ It is an extremely capable preservative in regards to its microbial coverage and its capacity to break cell-cell junctions on the corneal epithelium, thus allowing entry into the anterior chamber. ${ }^{45-47} \mathrm{BAK}$ as a polyquaternary ammonium compound acts as a detergent, which lyses cell membranes. ${ }^{48}$ Moreover, it remains in the eye long after its application as it incorporates itself into cell membranes for up to 7 days and has a half-life of 12 hours. ${ }^{49-51}$ Standard concentrations range from $0.015 \%$ to $0.02 \%$ BAK in ophthalmic solutions; however, toxicity has been demonstrated at concentrations as low as $0.005 \%$ BAK. ${ }^{52}$ There is, thus, reason to believe that such preservatives accumulate on the ocular surface as they are used at least once daily. As the preservative is collected on the ocular surface, prolonged exposure leads to toxicity. ${ }^{53}$ There are a slew of studies that investigate the effects of this preservative on the ocular surface. In vitro studies demonstrate the necrotic and apoptotic effects. ${ }^{54}$ Other studies show cell fragmentation, fibroblast proliferation, cell shrinkage, and cell death when tissue cultures are exposed to preservative alone and medications preserved with BAK. ${ }^{55}$ Antiglaucoma drops preserved with BAK are also strongly associated with topical inflammation. ${ }^{56}$ Several in vitro studies demonstrate a notable amount of toxicity to cells from the Chang and Wong-Kilbourne human conjunctival cell line, which are exposed to BAK, even at extremely low concentrations. ${ }^{57,58}$ When comparing the cytological differences of the conjunctiva of individuals using glaucoma medications and those who do not, there is a marked contrast. Those who have used antiglaucoma medications for even a short amount of time (14 days) display reduction in the number of goblet cells, epithelial keratinization, and squamous metaplasia. ${ }^{59,60}$ PGs have been especially scrutinized. Though they have a low systemic side effect profile, they are, as discussed earlier, infamous for the injection and inflammation with which they are associated, leading to poor adherence.

\section{PG analogues preserved with BAK}

Compared with other classes of antiglaucoma drugs, PG analogues have typically demonstrated less systemic side effects. Among the different formulations of $\mathrm{PG}$ 
analogues, the ocular side effect profiles are variable. One of the more commonly reported adverse effects is ocular hyperemia. ${ }^{34}$ To evaluate the association of these drugs and the widely reported ocular hyperemia, Guenoun et $\mathrm{al}^{61}$ evaluated the role of $3 \mathrm{PG}$ analogues on the expression of inflammatory markers using flow cytometry on a human conjunctival cell line. The results were compared with 3 controls including PGF2 $\alpha$, tumor necrosis factor $\alpha$ (TNF $\alpha$ ), and BAK at the 3 concentrations found in the commercial preparations of the PG analogues. PGF $2 \alpha$ did not demonstrate any induction effect. As predicted, TNF $\alpha$ induced all inflammatory markers at significantly higher levels than controls and BAK. BAK decreased the expression of the 2 adhesion molecules, likely due to the cytotoxicity as evidenced by DNA condensation evaluated by fluorocytometry. ${ }^{61}$ This is consistent with the widely known, dose-dependent deleterious cellular effects of BAK. ${ }^{57}$ The PG analogues also decreased the expression of inflammatory markers with latanoprost inducing the least amount of expression, followed by travoprost and then bimatoprost. Interestingly, the amount of inflammatory markers' expression negatively correlated with the concentration of BAK in each formulation. In other words, latanoprost has a higher concentration of BAK, but induced the least amount of inflammatory markers, which could be possibly due to the more immediate cytotoxicity than inflammation. Also, when this group evaluated for apoptosis, it was found that cell death also demonstrated a positive correlation with the concentrations of BAK in the $3 \mathrm{PG}$ analogues. This led to the conclusion that BAK was responsible for the inflammation and cellular toxicity that were associated with PG analogues. This may also explain the paradoxical reduction in ocular hyperemia seen with latanoprost compared with travoprost and bimatoprost. Compared with the trials of isolated BAK at concentrations found in commercial formulations, there was slightly more cell death and inflammation with BAK alone than with the commercial preparations, suggesting that the $\mathrm{PG}$ analogues have a protective role against the harmful effects of BAK. ${ }^{61}$ The role of PG cytoprotection was also introduced by a study, which compared latanoprost with $\beta$-blockers and BAK alone at concentrations found in the preserved preparations. Latanoprost again demonstrated less toxicity than the preserved $\beta$-blockers and solitary BAK at the same concentration, further suggesting a defensive role against preservative-induced damage. ${ }^{58}$

\section{Possible PG analogue cytoprotection from BAK}

In order to further investigate the cytoprotective mechanisms of PG analogues against BAK toxicity that were previously demonstrated, trials comparing the 3 leading PG analogues were completed. Using Chang cells and exposing them to the same PG analogues and similar concentrations of solitary BAK for 30 min, this group measured cell membrane compromise, cytosolic $\mathrm{H}_{2} \mathrm{O}_{2}$, cytosolic $\mathrm{O}_{2}$ free radicals, and apoptosis. As expected, solitary BAK demonstrated a dose-dependent effect. Latanoprost and travoprost were less detrimental than the corresponding BAK concentration. Both had significantly reduced amounts of $\mathrm{H}_{2} \mathrm{O}_{2}$ and proapoptotic effects, and latanoprost created less $\mathrm{O}_{2}$ radicals than the BAK counterpart. Bimatoprost did not, however, demonstrate this protective effect but demonstrated the least apoptotic effect as it had the lowest concentration of BAK among the 3. Based on this study, it appears that BAK causes oxidative effects that incite cytotoxicity, which is dose dependent. Although it appears that these PGF $2 \alpha$ analogues are antioxidative, PGFs are widely known to be proinflammatory. ${ }^{61,58}$ Perhaps the PGF analogues reduce the amount of generation of reactive oxygen species or induce other mechanisms of antioxidation or antiapoptosis; hence, this possibility could be further investigated. Another prospective explanation cited by the authors included the potential for BAK and the PG analogues forming an emulsion, thus reducing the BAK exposure. Though the mechanism for PG cytoprotection is not fully elucidated, the concept of PGF-related buffering of BAK-induced toxicity may have implications for its use. ${ }^{62}$

Despite the complexities of the interaction of BAK and PG analogues and the mechanism of medication-induced hyperemia and irritation, medications and their preservatives play a role in the development of OSD. ${ }^{38}$

\section{Consequences of chronic inflammation on surgical outcomes}

One of the most concerning effects of the subclinical inflammation caused by glaucoma medications is the failure of filtration surgery, which is often a last resort in the treatment of glaucoma. ${ }^{63,64}$ Success rates for trabeculectomy were almost 2 times greater for patients who used minimal glaucoma medications compared with those who used at least 2 medications. In addition, preoperative hypercellularity of chronic inflammatory cells (including fibroblasts, macrophages, and lymphocytes) of the trabecular meshwork was much lower for those who had successful surgeries in contrast to 
those whose surgeries failed. The presence of inflammatory cells may cause fibrosis of the bleb. Such data indicate a strong positive correlation between glaucoma medication and surgery failure. ${ }^{65}$ Several studies support the argument that filtration surgery is affected by the duration of use and the number of glaucoma medications being used by the patients. ${ }^{66-70}$ Preservative toxicity, specifically BAK toxicity, is suggested as a clear suspect in inducing this chronic and mild inflammatory response, which is strongly associated with detrimental surgery outcomes. ${ }^{71}$ Some investigators have suggested that there may be a trend toward alternative preservatives or preservative-free formulations in medical glaucoma treatment. ${ }^{66,72}$

\section{Options for alternative preservatives}

An interesting alternative option for preservatives is emulsions. Oil-in-water emulsions were developed several years ago as a possible alternative for treating dry eye associated with ophthalmic solutions. By positively charging the molecules using quaternary ammonium compounds, these emulsion solutions are attracted to the negatively charged ocular surface. ${ }^{73}$ This interaction provides increased amounts of time for the emulsion to remain on the ocular surface. BAK is comprised of several ammonium chlorides with different alkyl chain lengths. As both a preservative with toxic effects and a quaternary ammonium compound, which is positively charged, its excellent antimicrobial effectiveness is marred by its detrimental damage to the cells. BAK remains mostly in the lipophilic phase when prepared as an emulsion solution. ${ }^{74}$ Only $1.2 \%$ of BAK is the free and actively toxic preservative. ${ }^{75}$ Studies have shown that emulsions of BAK preservative reduce its detrimental effects, but they do not eliminate them. ${ }^{76}$

Although there are several other preservatives available, including phenylmercuric nitrate, chlorhexidine acetate, chlorobutanol, and polyquaternium-1, they have several disadvantages, including insufficient microbial coverage, being easily rendered inactive by common organic compounds or room temperatures, or causing similar detrimental effects of BAK on the ocular surface. Certain antimicrobials such as sorbate, which has almost no side effect profile, sodium perborate, which has a minimal side effect profile and very good microbial coverage, and stabilized oxychloro complex (SOC), which also provides broad spectrum of protection while being gentle, are other alternatives to BAK. SOC is an oxidant preservative, as opposed to detergent preservatives, is also known to disrupt cellular membranes, but only mildly. The selective bacteriocidal and fungicidal activities of oxidant preservatives are derived from their inherent ability to create oxidative stress, which eukaryotic cells are able to withstand through their antioxidant and enzymatic neutralizing agents. ${ }^{77} \mathrm{SOC}$ is currently available as a preservative for $0.1 \%$ brimonidine tartrate (Alphagan ${ }^{\circledR} \mathrm{P}$; Allergan, Irvine, California, USA), but the IOP-lowering capability is not as great as other medications. A newer preservative, sofZia is being used in preparations of travoprost. It is an ionic, buffered formula composed of zinc, borate, propylene glycol, and sorbitol. SofZia is effective in microbicidal activity for up to $99 \%$ of microorganisms after an 8-day incubation, and it meets the antimicrobial criteria of the United States Pharmacopeia. $^{78}$

\section{Efficacy and comfort of preservative-free non-PG ocular hypotensive medications}

Preservative-free brimonidine was among the first PG analogues available in a BAK-free preparation as brimonidine purite since 1996. Brimonidine purite is preserved with a SOC known as Purite ${ }^{\circledR}$. In this formulation, the concentration of brimonidine was lowered from $0.2 \%$ to $0.15 \%$. In randomized, double-masked trial, either brimonidine $0.2 \%$ or brimonidine purite was administered to 407 patients twice a day for 3 months. Both drugs were comparable in efficacy. The most common adverse event was hyperemia, which was reported less among participants who used brimonidine purite compared with brimonidine $0.2 \%$. The difference was not statistically significant. ${ }^{79}$ Another randomized, controlled, double-blind study performed for 1 year, however, did report that brimonidine purite was significantly more comfortable and safer for patients' use. ${ }^{80}$ Another medication available in a preservative-free formulation is timolol. One study found that the basal tear turnover, evaluated using computerized objective fluorophotometry, was, decreased by $30 \%$ in subjects using preserved timolol and increased approximately by $30 \%$ after subjects switched from preserved timolol to preservative-free timolol after 2 weeks compared with healthy controls. Participant inclusion criteria required subjects who used timolol plus BAK for at least 1 month before the trial began. TBUT values were decreased for both preserved and unpreserved timolol-treated eyes as compared with healthy controls. ${ }^{73}$ In another study where all patients were treated for at least 1 year with preserved $\beta$-blockers, unpreserved timolol, or 2 or more preserved medications, inflammatory markers in impression cytology samples exhibited a higher concentration of all markers, especially human leukocyte 
antigen. Immunoinflammatory marker interleukin-8 was more elevated among those using preserved medications rather than unpreserved timolol. ${ }^{81}$ There are several studies that also report similar findings in regards to preserved medications causing detrimental effects to the ocular surface compared with preservative-free medications. ${ }^{82,83}$

\section{Preservative-free PG analogues}

Recently, an effort has been made to create preservative-free solutions for antiglaucoma medications. PG analogues have come into favor among ophthalmologists as they are the most effective drugs for the treatment of ocular hypertension ${ }^{84}$ and maintain fewer systemic side effects compared with older $\beta$-blockers, miotics, and adrenergic agonists. ${ }^{85}$ Generally, bimatoprost, latanoprost, and travoprost are the PG analogues available. Studies comparing the efficacy of these drugs show that these 3 PG analogues along with timolol are the most effective drugs at lowering IOP compared with most other classes of ocular hypotensives being used today. ${ }^{12}$ Reports of side effect profiles of the $3 \mathrm{PG}$ analogues available differ; however, latanoprost is usually associated with less hyperemia. ${ }^{86,87}$ Currently, travoprost is the only preservative-free PG analogue available. In the following discussion, BAK-free travoprost is reviewed.

\section{Travoprost BAK-free}

Travoprost BAK-free solution (Travatan $Z^{\circledR}$; Alcon Laboratories, Inc, Fort Worth, Texas, USA) is the first PG analogue without BAK preservative made for commercial use. It is preserved with sofZia, which, as previously described, is an ionic buffered system. The efficacy of travoprost with and without BAK is demonstrated to be significantly equal in regards to the ability to lower IOP among 661 patients diagnosed with open-angle glaucoma or ocular hypertension over a period of 3 months, with $95 \%$ confidence intervals within $\pm 0.8 \mathrm{~mm} \mathrm{Hg}$. Within this study, treatment-related ocular hyperemia was reported $9.0 \%$ of the time among those using travoprost preserved with $0.004 \%$ BAK and $6.4 \%$ of the time among those using BAK-free travoprost, but these data were not statistically significant. Observable change from baseline in regards to change in visual acuity, ocular signs of inflammation, dilated fundus examinations, and visual fields was not worrisome in either group, indicating that the BAK-preserved and BAK-free formulation have tolerable safety assessments. This study did not evaluate patients' comfort. Attrition rates due to treatment-related side effects were recorded to be $1.5 \%$ in travoprost BAK-free and $1.2 \%$ in travoprost $0.004 \% \mathrm{BAK}^{88}$
A recently reported double-blind, randomized, controlled trial compared the evaluation of patients' comfort after a single dose of BAK-free travoprost $0.004 \%$ in 1 eye and latanoprost $0.005 \%$ in the other eye. Patients underwent a 3-day washout period during which patients did not use any glaucoma medications to allow ocular surface epithelial turnover. One drop of medicine was administered in one eye. Comfort was measured by a baseline visual analogue scale (VAS) given at every 5 seconds for first minute and then at $2,5,10,15,20$, and 30 minutes. After this was completed, 1 drop of the other medicine was administered in the opposite eye and the VAS was completed as with the first eye. Results from the completed VAS demonstrated no difference in comfort level after 5 seconds of eye-drop administration, and the average comfort ratings were comparable with controlled untreated subjects. Mean levels of peak discomfort were also similar, but the average time needed to return to baseline levels of comfort was higher for those using latanoprost than for those using BAK-free travoprost; however, this was not significant. A comfort survey completed by the study subjects revealed no difference between the 2 drugs in regards to burning, stinging, or foreign body sensation. Participants favored latanoprost $31 \%$ of the time and BAKfree travoprost $33 \%$ of the time, while they reported no preference $35 \%$ of the time. Though this study importantly demonstrates no significant differences in comfort between BAK-free travoprost and latanoprost preserved with BAK, the data collected were after only 1 dose. If the medications were given chronically, a more clinically meaningful conclusion regarding adverse effects of BAK-preserved and BAK-free medications in the anterior segment of the eye could have been made. ${ }^{89}$ In vitro studies compared the differing effects of 30-minute exposure to travoprost BAK-free with preserved travoprost, commercially available latanoprost, phosphate buffer saline (PBS) control, and BAK alone at concentrations found in commercial formulations on the Wong-Kilbourne conjunctiva-derived cell line. The outcome measurements used included assays for cell viability, cellular proliferation, membrane integrity, and cytotoxicity. All measures were elevated in cells exposed to preserved latanoprost and BAK alone. There was no difference in cells exposed to PBS control and BAK-free travoprost. ${ }^{90}$

Another group used human corneal epithelial cell culture systems to demonstrate the effect of preserved latanoprost compared with BAK preservative-free travoprost. For controls, this group used lubricant eye drops, 70\% methanol, and $0.3 \%$ gentamicin sulfate; the latter 2 are known to be cytotoxic. Measurements were made using fluorescent 
molecular probes, one of which reacts with esterase in live cells and one of which crosses through compromised cell membranes. The cells were incubated in undiluted medications for 25 minutes and were then evaluated. Results were reported as percentages of the live controls. Of the cells exposed to BAK-free travoprost, approximately $56.2 \%$ were viable, whereas in those exposed to latanoprost only approximately $5.1 \%$ were viable. The percentage of cells viable after latanoprost exposure was not very different than those exposed to the dead controls; this was, however, not statistically significant. ${ }^{91}$

In vitro studies may not accurately reflect the effect of medication on the ocular surface as the physiologic conjunctival environment cannot be re-created in the laboratory setting. One criticism of using in vitro studies, particularly of the human corneal epithelial cell culture system - monolayer, is that results from these cells do not accurately reflect the cornea's ability to tolerate effects of preservatives. In comparison, stratified cell layers expressing corneal keratin are shown to provide an effective substrate on which to perform in vitro studies with more realistic results. ${ }^{92}$ Khoh-Reiter and Jessen used the 3-dimensional corneal epithelial cultures, which showed that this system expressed 3 of the tested corneal epithelial markers more than in the monolayer cell system; they compared the effect of latanoprost preserved with $0.02 \% \mathrm{BAK}$, olopatadine (0.01\% BAK), and BAK-free travoprost after exposure for 10 and 25 minutes. Concomitantly, this research group also instilled commercially available latanoprost or its vehicle containing $0.02 \%$ BAK in monkey eyes and performed pachymetry at regular intervals for 1 year. Corneal thickness is suggestive of ocular inflammation secondary to edema. ${ }^{93}$ Among cells exposed to test solutions, only those exposed to positive controls demonstrated decreased cell viability after 10 minutes of exposure. After 25 minutes, cell viability decreased significantly in cell cultures exposed to latanoprost. The authors of this study noted that there was no significant difference among the decreases in viability after 25 minutes between latanoprost and olopatadine when each was compared with BAK-free travoprost. In the pachymetry arm, study monkey corneas that were exposed to a twice-daily $30 \mu \mathrm{L}$ dosing of latanoprost in 1 eye and vehicle with $0.02 \%$ BAK in the other were compared with control monkey corneas, where vehicle with $0.02 \%$ BAK was used in 1 eye and the other was not treated. Results did not show histological signs of ocular irritation or a significant difference in corneal thickness between the study group and the controls. ${ }^{94}$
Though in vitro studies have very clear limitations, they do provide a description of the effects of these drugs on certain cell types relative to the other drugs being evaluated. It is, thus, very clear that travoprost BAK-free is less or at least equally detrimental to these conjunctival cells in relation to commercially available latanoprost. Perhaps the physiologic ocular surface attenuates or exacerbates this relationship.

An in vivo model used to study these medications is the animal model. Often, rabbits' eyes are used. Whitson et al ${ }^{95}$ evaluated the effects of travoprost without BAK compared with latanoprost after administering a 3-minute bath in 1 eye of New Zealand rabbits and then 1 drop/min for 3 minutes in the other eye. In vivo confocal microscopy of the corneas immediately after exposure revealed no changes in cell shape or structure in eyes exposed to travoprost BAK-free as compared with smaller, brighter cells with irregular borders noted on the corneas of eyes exposed to commercially prepared (preserved with BAK) latanoprost. Differences were statistically significant. ${ }^{95}$ This study is helpful in that it uses a live animal model rather than in vitro testing, and this dosing regimen model allows investigators to quickly establish whether an immediate toxic effect is elicited. One may argue that the prolonged exposure time does not simulate a normally prescribed dosing regimen for patients. Much of the studies of Travatan $\mathrm{Z}$ that are available utilize cells, tissue, or in vivo exposure to test solutions for greater amounts of time than human eyes. In clinical settings, drops reside on the ocular surface for an average of 2.9 seconds. Many investigators inoculate substrate tissue for many more seconds to even minutes in order to demonstrate possible toxic effects quickly. Thus, many effects that these studies describe may not be a true extrapolation to the effects on patients' eyes. However, BAK remains on the ocular surface as it is a detergent, which incorporates itself into cell membranes. ${ }^{96}$ There is, thus, a reason to believe that the exposure time in laboratory settings could actually be shorter than in clinical settings. One study that dosed rabbits' eyes in a once-a-day fashion as in the clinical setting also reported similar findings. Investigators treated the rabbits' eyes once per day for 30 days and then killed the animals. Conjunctival tissue was plated, and masked evaluators counted lymphocytes to measure the degree of inflammation. Corneas were also viewed under an electron microscope, and masked physicians graded the quality in regards to microvilli changes. Eyes treated with travoprost and artificial tears demonstrated significantly lower inflammatory cell infiltration compared with latanoprost with BAK. There was no significant difference in eyes treated with travoprost vs artificial tears. 
In addition, eyes that were subjected to latanoprost preserved with BAK demonstrated a fibroblast infiltration, which was not seen in eyes treated with travoprost. Corneal changes as measured by qualitative grading of microvilli were also significantly different between travoprost and latanoprost. Again, differences seen between travoprost and artificial tears were not significant. ${ }^{97}$ These results are important as they exhibit changes on the ocular surface after eyes were exposed to therapeutic doses for an extended period of time. Moreover, the results are consistent with in vitro findings that exhibit the detrimental effects of BAK, but at prolonged exposure and incubation times.

As with in vitro study models, animal models also have their limitations that are notable. Several studies performed on rabbits' eyes are scrutinized for using rabbits as models for measuring toxicity. One study indicates that rabbits' eyes are more prone to corneal irritation ${ }^{98}$ and are susceptible to irritant effects for longer periods of time. ${ }^{99}$ Though there are no perfect animal models, these types of studies serve the purpose to evaluate medications in a physiological environment.

A randomized, controlled, double-blind study enrolled 109 human subjects who had glaucoma or ocular hypertension and who underwent a washout period specific for their previous medications. They were then given either travoprost or Travatan $\mathrm{Z}$ for 2 weeks, which is an established time period for travoprost to optimize IOP. ${ }^{100}$ Participants' IOPs were measured on the 14 th and 15 th day at $8 \mathrm{AM}$ and $8 \mathrm{PM}$, and at 36, 48, and 60 hours post last dose. Patient-reported and physician-observed adverse events were also recorded. The 2 medications cause similar and statistically significant lowering of IOP at all times measured, implying that BAK does not hinder ocular hypotensive effects and that travoprost preserved with sofZia is efficacious. The adverse-event profile was also similar, Travatan $Z$ being associated with 3 reports of either dry eye or hyperemia and travoprost with BAK being associated with 4 reports. ${ }^{101}$

It is well known to practicing ophthalmologists that certain individuals react to BAK-preserved formulations with severe hyperemia and ocular surface inflammation, whereas others do not demonstrate a response. It is, thus, important to recognize that "BAK-sensitive" individuals are important to evaluate when considering the effects of BAK preserved vs BAK-free ophthalmic solutions so that clinicians can optimize treatments for their patients. One study enrolled patients who were found to have a decreased tolerability to traditionally preserved $\mathrm{PG}$ analogues. All patients were using either latanoprost or bimatoprost for at least 1 week and then were asked to discontinue and start using Travatan $\mathrm{Z}$ once each morning for 12 weeks. Outcome measurements were conducted using OSD index (OSDI) score comparison, Goldmann applanation tonometry, conjunctival hyperemia grading, Snellen visual acuity, and slit lamp biomicroscopy. OSDI scores were an average of 8.7 for travoprost BAK-free, 12.0 for latanoprost, and 13.2 for bimatoprost. Interestingly, patients who, on visit 1 , demonstrated more severe reactions to their original medications showed a greater reduction in OSDI scores than those who demonstrated more mild symptoms initially, though all patients reported some reduction in OSDI score after using travoprost BAK-free. Remarkably, of the patients who reported initial severe symptoms, 25\% reported "normal" after switching to the preservative-free formulation. IOP decreased in those who switched from latanoprost but not for those who switched from bimatoprost. Hyperemia and visual acuity were significantly reduced and improved, respectively, with the use of travoprost BAK-free. Adverse events were still reported with the use of travoprost, including conjunctival hyperemia in $6 \%$ and a small change in visual acuity in $4 \%$ of participants. Among the participants, $72 \%$ reported that they preferred preservative-free travoprost to their previous medication. ${ }^{102}$ Though these results show an impressive improvement after switching to BAK-free PG analogue formulations, it is important to note that this trial did not have a control group and the participants were, thus, not masked. As every participant's symptoms improved with BAK-free preparations, a masked, double-blind, randomized, controlled trial evaluating these parameters is warranted.

\section{Conclusion}

Primary open-angle glaucoma is expected to be a significant contributing factor to the increasing rates of irreversible blindness. As the prevalence of this disease is expected to increase in the upcoming years, it is important that medical therapy be optimized to prevent the progression of the pathologic process and maximize the safety profiles to ensure patients' comfort. Medical treatment is effective if administered properly, but comfort concerns often cause patients to neglect taking their medications. Reduced comfort and consequent reduced adherence to dosing regimens are widely attributed to the preservative used in most glaucoma medications. BAK is repeatedly implicated in the ocular surface adverse events that are reported. As PG analogues cause minimal systemic side effects, they are preferred by patients and physicians. The main side effect profile includes ocular hyperemia and inflammation. In light of the well-documented association of BAK and OSD, BAK-free PG formulations 
should be expected to demonstrate an even smaller side effect profile. Given the far-reaching effects of ocular inflammation in regards to adherence and failure of filtration surgery, this advancement appears to be much needed.

Several studies, as delineated here, illustrate that the new BAK-free PG analogue, Travatan $\mathrm{Z}$, demonstrates similar efficacy, even up to 60 hours after the last dose once the drug is at therapeutic levels, and reduced amounts of cellular toxicity. Upon reviewing the literature, it is evident that more randomized, controlled, double-masked studies are needed to evaluate the expected improved safety profile for this medication. In the randomized, controlled, double-blind study completed by Gross et al, ${ }^{101}$ patients used the medications for only 2 weeks. Perhaps a longer time period will illicit a difference in patients' comfort between travoprost preserved with BAK and Travatan Z. In the randomized controlled trial by Henry et al, ${ }^{102}$ one of the inclusion criteria was that patients were having ocular irritation while using BAK-preserved medications before the trial initiation. This is important to note as not all patients are as sensitive to the adverse reaction as described with BAK use. It is, therefore, of interest to evaluate Travatan $\mathrm{Z}$ in those patients who are inclined to react unfavorably. Having a preservative-free PG analogue as an option for glaucoma treatment will allow for more personalized and effective patient care. If a drug regimen that does not conflict with patients' well-being or decrease their quality of life can be established, the treatment of glaucoma will be significantly enhanced. Further investigation of Travatan $\mathrm{Z}$ may yield promising results.

\section{Disclosure}

The authors neither have affiliation to any pharmaceutical company nor have any conflict of interest. Both work in an academic setting in public clinics for the University of Virginia in Charlottesville, Virginia, USA.

\section{References}

1. Quigly HA, Broman AT. The number of people with glaucoma worldwide in 2010 and 2020. Br J Ophthalmol. 2006;90(3):262-267.

2. Bourne RRA. Worldwide glaucoma through the looking glass. $\mathrm{Br}$ Ophthalmol. 2006;90(3):253-254.

3. Mendrinos E, Shaarawy T. Glaucoma surgery: toward a new global vision. Expert Rev Ophthalmol. 2007;2:149-153.

4. World Health Organization. Prevention of blindness and visual impairment. Available from: http://www.who.int/blindness/causes/priority/en/ index7.html

5. Heijl A, Leske MC, Bengtsson B, Hyman L, Bengtsson B, Hussein M. Reduction of intraocular pressure and glaucoma progression: results from the Early Manifest Glaucoma Trial. Arch Ophthalmol. 2002;120: 1268-1279.

6. Friedman DS, Wolfs RC, O'Colmain BJ, et al. Prevalence of open-angle glaucoma among adults in the United States. Arch Ophthalmol. 2004;122:532-538.
7. Friedman DS, Jampel HD, Monoz B, et al. The prevalence of openangle glaucoma among blacks and whites 73 years or older: the Salisbury Eye Evaluation Glaucoma Study. Arch Ophthalmol. 2006;124: $1525-1630$.

8. Lee PP, Walt JG, Doyle JJ, et al. A multicenter, retrospective pilot study of resource use and costs associated with severity of disease in glaucoma. Arch Ophthalmol. 2006;124:12-19.

9. Kass MA, Heuer DK, Higginbotham EJ, et al. The ocular hypertension treatment study. Arch Ophthalmol. 2002;120(6):701-713.

10. Collaborative Normal-Tension Glaucoma Study Group. Comparison of glaucomatous progression between untreated patients with normaltension glaucoma and patients with therapeutically reduced intraocular pressure. Am J Ophthalmol. 1998;126:487-497.

11. Lichter PR, Musch DC, Gillespie BW, et al. Interim clinical outcomes in the Collaborative Initial Glaucoma Treatment Study (CIGTS) comparing initial treatment randomized to medications or surgery. Ophthalmology. 2001;108:1943-1953.

12. van der Valk R, Webers CA, Schouten JS, Zeegers MP, Hendrikse F, Prins MH. Intraocular pressure-lowering effects of all commonly used glaucoma drugs: a meta-analysis of randomized clinical trials. Ophthalmology. 2005;112:1177-1185.

13. Lim KS, Nau CB, O’Byrne MM, et al. Mechanism of action of bimatoprost, latanoprost, and travoprost in healthy subjects: a crossover study. Ophthalmology. 2008;115(5):790-795.

14. Konstas AGP, Kozobolis VP, Katsimpris I, et al. Efficacy and safety of latanoprost versus travoprost in exfoliative glaucoma patients. Ophthalmology. 2007;114(4):653-657.

15. Ali FS, Akpek EK. Glaucoma and dry eye. Ophthalmology. 2009; 116(6):1232.

16. Shaumberg DA, Sullivan DA, Buring JE, et al. Prevalence of dry eye syndrome among US women. Am J Ophthalmol. 2003;136:318-326.

17. Moss SE, Klein R, Klein BE. Prevalence of and risk factors for dry eye syndrome. Arch Ophthalmol. 2000;118:1264-1268.

18. McCarty CA, Bansal AK, Livingston PM, et al. The epidemiology of dry eye in Melbourne, Australia. Ophthalmology. 1998;105:1114-1119.

19. Schein OD, Hochberg MC, Munoz B, et al. Dry eye and dry mouth in the elderly. Arch Intern Med. 1999;159:1359-1363.

20. Doughty MJ, Fonn D, Richeter D, et al. A patient questionnaire approach to estimating the prevalence of dry eye symptoms in patients presenting to optometric practices across Canada. Optom Vis Sci. 1997;74:624-631.

21. Lin PY, Tsai SY, Cheng CY, et al. Prevalence of dry eye among an elderly Chinese population in Taiwan: the Shihpai Eye Study. Ophthalmology. 2003;110:1096-1101.

22. Lee AJ, Lee J, Saw S-M, et al. Prevalence and risk factors associated with dry eye symptoms: a population based study in Indonesia. $\mathrm{Br} J$ Ophthalmol. 2002;86:1347-1351.

23. Shimmura S, Shimazaki J, Tsubota K. Results of a population-based questionnaire on the symptoms and lifestyles associated with dry eye. Cornea. 1999;18:408-411.

24. Schiffman RM, Walt JG, Jacobsen G, et al. Utility assessment among patients with dry eye disease. Ophthalmology. 2003;110:1412-1419.

25. Clegg JP, Guest JF, Lehman A, Smith AF. The annual cost of dry eye syndrome in France, Germany, Italy, Spain, Sweden, and the UK. Ophthalmic Epidemiol. 2006;13:263-274.

26. Perry HD. Dry eye disease: pathophysiology, classification, and diagnosis. Am J Manag Care. 2008;14 Suppl 3:S79-S87.

27. Schmier JK, Covert DW. Characteristics of respondents with glaucoma and dry eye in a national panel survery. Clin Ophthalmol. 2009;3:645-650.

28. Rossi GC, Tinelli C, Pasinetti GM, et al. Dry eye syndrome-related quality of life in glaucoma patients. Eur J Ophthalmol. 2009;19(4):572-579.

29. Nordmann JP, Auzanneau N, Ricard S, Berdeaux G. Vision-related quality of life and topical glaucoma treatment side-effects. Health Qual Life Outcomes. 2003;1:75.

30. Malvitte L, Montange T, Vejux A, et al. Measurement of inflammatory cytokines by multicytokine assay in tears of patients with glaucoma topically treated with chronic drugs. Br J Ophthalmol. 2007;91: 29-32. 
31. Schwartz GF, Tan J, Kotak S. Hyperemia-associated costs of medication changes in glaucoma patients treated initially with prostaglandin analogs. J Ocul Pharmacol Ther. 2009;25(6):555-561.

32. Leung EW, Medeiros FA, Weinreb RN. Prevalence of ocular surface disease in glaucoma patients. J Glaucoma. 2008;17:350-355.

33. Friedman DS, Hahn SR, Gelb L, et al. Doctor-patient communication, health-related beliefs, and adherence in glaucoma results from the Glaucoma Adherence and Persistency Study. Ophthalmology. 2008;115: 1320-1327.

34. Feldman RM. Conjunctival hyperemia and the use of topical prostaglandins in glaucoma and ocular hypertension. J Ocul Pharmacol Ther. 2003;19:23-35.

35. Rees G, Leong O, Crowston JC, Lamoureux EL. Intentional and unintentional nonadherence to ocular hypotensive treatment in patients with glaucoma. Ophthalmology. 2010;117(5):903-908.

36. Schwartz GF. Compliance and persistency in glaucoma follow-up treatment. Current Opin Ophthalmol. 2005;16(2):114-121.

37. Parrish RK, Palmberg P, Sheu WP; for XLT Study Group. A comparison of latanoprost, bimatoprost, and travoprost in patients with elevated intraocular pressure: a 12-week, randomized, masked-evaluator multicenter study. Am J Ophthalmol. 2003;135(5):688-703.

38. Broadway DC, Chang LP. Trabeculectomy, risk factors for failing and the preoperative state of the conjunctiva. J Glaucoma. 2001;10: 237-249.

39. Pisella PJ, Pouliquen P, Baudouin C. Prevalence of ocular symptoms and signs with preserved and preservative-free glaucoma medication. Br J Ophthalmol. 2002;86:418-423.

40. Jaenen N, Baudouin C, Pouliquen P, et al. Ocular symptoms and signs with preserved and preservative-free glaucoma medications. Eur $J$ Ophthalmol. 2007;17:341-349.

41. Rahman MQ, Tejwani D, Wilson JA, Butcher I, Ramaesh K. Microbial contamination of preservative free eye drops in multiple application containers. Br J Ophthalmol. 2006;90:139-141.

42. Schein OD, Hibberd PL, Starch T, Baker AS, Kenyan KR. Microbial contamination of the in-use ocular medications. Arch Ophthalmol. 1992;110:82-85

43. Geyer O, Bottone EJ, Podos SM, Schumer RA, Asbell PA. Microbial contamination of medications used to treat glaucoma. Br J Ophthalmol. 1995;79:376-379.

44. Whitson JT, Varner DL, Grove D, Netland PA. Glaucoma drugs and the ocular surface. Rev Ophthalmol. 2006;13(11):69-79.

45. Burnstein NL, Klyce SD. Electrophysiologic and morphologic effects of ophthalmic preparations on rabbit cornea epithelium. Invest Ophthalmol Vis Sci. 1977;6:899-911.

46. Gasset AR, Ishii Y, Kaufman HE, Miller T. Cytotoxicity of ophthalmic preservatives. Am J Ophthalmol. 1974;7(1):98-105.

47. Berdy GJ, Abelson MB, Smith LM, George MA. Preservative-free artificial tear preparations. Assessment of corneal epithelial toxic effects. Arch Ophthalmol. 1992;110:528-532.

48. Tripathi BJ, Tripathi RC, Killi SP. Cytotoxicity of ophthalmic preservatives on human corneal epithelium. Lens Eye Toxic Res. 1992;9(3-4): 361-375.

49. Burstein NL. The effects of topical drugs and preservatives on the tears and corneal epithelium in dry eye. Trans Ophthalmol Soc $U K$ 1985;104:402-409.

50. Shaw AJ, Balls M, Clothier RH, Bateman ND. Predicting ocular irritancy and recovery from injury using Madin-Darby canine kidney cells. Toxicol In Vitro. 1991;5:169-174.

51. Champeau EJ, Edelahuser HF. Effect of ophthalmic preservatives on the ocular surface: conjunctival and corneal uptake and distribution of benzalkonium chloride and chlorhexidine digluconate. In: Holly FJ, editor. The Preocular Tear Film. Lubbock, TX: Dry Eye Institute, Inc; 1986.

52. Ichijima H, Petroll WM, Jester JV, Cavanagh HD. Confocal microscopic studies of living rabbit cornea treated with benzalkonium chloride. Cornea. 1992;11:221-225.
53. Yee RW. The effect of drop vehicle on the efficacy and side effects of topical glaucoma therapy: a review. Current Opin Ophthalmology. 2007;18:134-139.

54. de Saint Jean M, Brignole F, Bringuier AF, Bauchet A, Felmann G, Bauduoin C. Effects of benzalkonium chloride on growth and survival of Chang conjunctival cells. Invest Ophthalmol Vis Sci. 1999;40: 619-630.

55. Williams DE, Nguyen KD, Shapourifar-Tehrani S, Kitada S, Lee DA. Effects of timolol, betaxolol, and levobunolol on human tenon's fibroblasts in tissue culture. Invest Ophthalmol Vis Sci. 1992;33: 2233-2241.

56. Baudouin C, Pisella PJ, Fillacier K, et al. Ocular surface inflammatory changes induced by topical antiglaucoma drugs. Ophthalmology. 1999;106(3):556-563

57. Debbasch C, Brignole F, Pisella PJ, et al. Quaternary ammoniums and other preservatives' contribution in oxidative stress and apoptosis on Chang conjunctival cells. Invest Ophthalmol Vis Sci. 2001;42: 642-652.

58. Pisella PJ, Debbasch C, Hamard P, et al. Conjunctival proinflammatory and proapoptotic effects of latanoprost and preserved and unpreserved timolol: an ex vivo and in vitro study. Invest Ophthalmol Vis Sci. 2004;45:1360-1368

59. Costagliola $C$, Prete AD, Incorvaia $C$, et al. Ocular surface changes induced by topical application of latanoprost and timolol: a short-term study in glaucomatous patients with and without allergic conjunctivitis. Graefes Arch Clin Exp Ophthalmol. 2001;239:809-814.

60. Liesegang TJ. Conjunctival changes associated with glaucoma therapy: implications for the external disease consultant and treatment of glaucoma. Cornea. 1998;17:574-583.

61. Guenoun JM, Baudouin C, Rat P, et al. In vitro study of inflammatory potential and toxicity profile between latanoprost, travoprost, and bimatoprost on conjunctiva-derived epithelial cells. Invest Ophthalmol Vis Sci. 2005;46:2444-2450.

62. Guenoun JM, Baudouin C, Rat P, et al. In vitro comparison of cytoprotective and antioxidative effects of latanoprost, travoprost, and bimatoprost on conjunctiva-derived epithelial cells. Invest Ophthalmol Vis Sci. 2005;40:4594-4599.

63. Skuta GL, Parrish RK II. Wound healing in glaucoma filtering surgery. Surv Ophthalmol. 1987;80:624-627.

64. Hitchings RA, Grierson I. Clinico-pathologic correlation in eyes with failed fistulizing surgery. Trans Ophthalmol Soc $U K$. 1983;103:84-88.

65. Broadway DC, Grierson I, O'Brien C, Hitchings RA. Adverse effects of topical antiglaucoma medication, II: the outcome of filtration surgery. Arch Ophthalmol. 1994;112:1446-1454.

66. Kuppens EV, de Jong CA, Stolwijk TR, de Kreizer RJ, van Best JA. Effects of timolol with and without preservative on the basal tear turnover in glaucoma. Br J Ophthalmol. 1993;77:590-596.

67. Baudouin C. Detrimental effect of preservatives in eyedrops: implications for the treatment of glauocoma. Acta Ophthalmol. 2008;86:716-726.

68. Young TL, Higginbotham EJ, Zou X, Farber MD. Effects of topical glaucoma drugs on fistulized rabbit conjunctiva. Ophthalmology. 1990;97:1423-1427.

69. Lavin MJ, Wormald RPL, Migdal CS, Hitchings RA. Influence of prior therapy on the success of trabeculectomy. Arch Ophthalmol. 1990;108:1543-1548.

70. Johnson DH, Yoshikawa K, Brubaker RF, Hodge DO. The effect of long-term medical therapy on the outcome of filtration surgery. Am J Ophthalmol. 1994;117:139-148.

71. Baudouin C. Mechanisms of failure in glaucoma filtering surgery: a consequence of antiglaucomatous drugs? Int J Clin Pharmacol Res. 1996;16:29-41.

72. Baudouin C. Side effects of antiglaucomatous drugs on the ocular surface. Curr Opin Ophthalmol. 1996;7:80-86.

73. Tamilvanan S, Benita S. The potential of lipid emulsion for ocular delivery of lipophilic drugs. Eur J Pharm Biopharm. 2004;58: 357-368. 
74. Sila-On W, Vardhanabhuti N, Ongpipattanakul B, Kulvanich P. The influence of physicochemical properties of preservative compounds on their distribution into various phases of oil in water submicron emulsion. PDA J Pharm Sci Technol. 2006;60:172-181.

75. Sznitowska M, Janicki S, Dabrowska EA, Gajewska M. Physicochemical screening of antimicrobial agents as potential preservatives for submicron emulsions. Eur J Pharm Sci. 2002;15:489-495.

76. Liang H, Brignole-Baudouin F, Rabinovich-Guilatt L, et al. Reduction of quaternary ammonium-induced ocular surface toxicity by emulsions: an in vivo study in rabbits. Mol Vis. 2008;14:204-216.

77. Kaur IP, Lal S, Rana C, Kakkar S, Singh H. Ocular preservatives: associated risks and newer options. Cutan Ocul Toxicol. 2009;28(3): 93-103.

78. Kahook MY. Travoprost Z ophthalmic solution with sofZia: clinical safety and efficacy. Expert Rev Ophthalmol. 2007;2:363-368.

79. Mundorf T, Williams R, Whitcup S, et al. A 3-month comparison of efficacy and safety of brimonidine-purite $0.15 \%$ and brimonidine $0.2 \%$ in patients with glaucoma or ocular hypertension. J Ocul Pharmacol Ther. 2003;19(1):37-44.

80. Katz LJ. Twelve-month evaluation of brimonidine-purite versus brimonidine in patients with glaucoma or ocular hypertension. J Glaucoma. 2002;11(2):119-126.

81. Baudouin C, Hamard P, Liang H, et al. Conjunctival epithelial cell expression of interleukins and inflammatory markers in glaucoma patients treated over the long term. Ophthalmology. 2004;111(12): 2186-2192.

82. Ishibashi T, Yokoi N, Kinoshita S. Comparison of the short-term effects on the human corneal surface of topical timolol maleate with and without benzalkonium chloride. J Glaucoma. 2003;12:486-490.

83. Baudouin C, de Lunardo C. Short term comparative study of topical $2 \%$ carteolol with and without benzalkonium chloride in healthy volunteers. Br J Ophthalmol. 1998;82:39-42.

84. Vass C, Hirn C, Sycha T, et al. Medical interventions for primary open angle glaucoma and ocular hypertension. Cochrane Database Syst Rev. 2007;(4):CD003167.

85. Hodge WB, Lachaine J, Steffensen I, et al. The efficacy and harm of prostaglandin analogues for IOP reduction in glaucoma patients compared to dorzolamide and brimonidine: a systematic review. $\mathrm{Br} \mathrm{J}$ Ophthalmol. 2008;92:7-12.

86. Eyawo O, Nachega J, Lefebvre P, et al. Efficacy and safety of prostaglandin analogues in patients with predominately primary open-angle glaucoma or ocular hypertension: a meta-analysis. Clin Ophthalmol. 2009;3:447-456.

87. Aptel F, Cucherat M, Denis P. Efficacy and tolerability of prostaglandin analogs: a meta-analysis of randomized controlled clinical trials. J Glaucoma. 2008;17(8):667-673.

88. Lewis RA, Katz GJ, Weiss MJ, et al. Travoprost $0.004 \%$ with and without benzalkonium chloride: a comparison of safety and efficacy. J Glaucoma. 2007;16(1):98-103.
89. Godfrey DA, Peplinski LS, Stewart JA, Stewart WC. A comfort comparison of travoprost BAK-free $0.004 \%$ versus lantanoprost $0.005 \%$ in patients with primary open-angle glaucoma or ocular hypertension. Clin Ophthalmol. 2009;3:189-194.

90. Baudouin C, Riancho L, Warnet JM, Brignole F. In vitro studies of antiglaucomatous prostaglandin analogues: travoprost with and without benzalkonium chloride and preserved latanoprost. Invest Ophthalmol Vis Sci. 2007;48(9):4123-4128.

91. Yee R, Norcom EG, Zhao XC. Comparison of the relative toxicity of travoprost $0.004 \%$ without benzalkonium chloride and latanoprost $0.005 \%$ in an immortalized human cornea epithelial cell culture system. Adv Ther. 2006;23:511-518.

92. Nguyen DH, Beuerman RW, de Wever B, Rosdy M. Three-dimensional construct of the human corneal epithelium for in vitro toxicology. In: Salem H, Katz SA, editors. Alternatives Toxicological Methods. Boca Raton, Florida: CRC Press; 2003:147-159.

93. Morgan RL, Sorenson SS, Castles TR. Prediction of ocular irritation only corneal pachymetry. Food Chem Toxicol. 1987;25:609-613.

94. Khoh-Reiter S, Jessen BA. Evaluation of the cytotoxic effects of ophthalmic solutions containing benzalkonium chloride on corneal epithelium using an organotypic 3-D model. BMC Ophthalmol. 2009;9:5.

95. Whitson JT, Cavanagah HD, Lakshman N, Petroll WM. Assessment of corneal epithelial integrity after acute exposure to ocular hypotensive agents preserved with and without benzalkonium chloride. Adv Ther. 2006;23:663-671.

96. Green K, Jack MC, Cheeks L, et al. Detergent penetration into young and adult rabbit eyes: comparative pharmacokinetics. Cutan Ocul Toxicol. 1987;6:89-107.

97. Kahook MY, Noecker RJ. Comparison of corneal and conjunctival changes after dosing of travoprost preserved with sofZia, latanoprost with $0.02 \%$ benzalkonium chloride, and preservative-free artificial tears. Cornea. 2008;27:339-343.

98. Curren RD, Harbell JW. In vitro alternatives for ocular irritation. Environ Health Perspect. 1998;106:485-492.

99. Roggeband R, York M, Pericoi M, Braun W. Eye irritation responses in rabbit and man after single applications of equal volumes of undiluted model liquid detergent products. Food Chem Toxicol. 2000;38: 727-734.

100. Netland PA, Landry T, Sullivan EK, et al; for Travoprost Study Group. Travoprost compared with latanoprost and timolol in patients with open-angle glaucoma or ocular hypertension. Am J Ophthalmol. 2001;132:472-484.

101. Gross RL, Peace JH, Smith SE, et al. Duration of IOP reduction with travoprost BAK-free solution. J Glaucoma. 2008;17:217-222.

102. Henry JC, Peace JH, Stewart JA, Stewart WC. Efficacy, safety, and improved tolerability of travoprost BAK-free ophthalmic solution compared with prior prostaglandin therapy. Clin Ophthalmol. 2008;2:613-621.
Clinical Ophthalmology

\section{Publish your work in this journal}

Clinical Ophthalmology is an international, peer-reviewed journal covering all subspecialties within ophthalmology. Key topics include: Optometry; Visual science; Pharmacology and drug therapy in eye diseases; Basic Sciences; Primary and Secondary eye care; Patient Safety and Quality of Care Improvements. This journal is indexed on Submit your manuscript here: http://www.dovepress.com/clinical-ophthalmology-journal

\section{Dovepress}

PubMed Central and CAS, and is the official journal of The Society of Clinical Ophthalmology (SCO). The manuscript management system is completely online and includes a very quick and fair peer-review system, which is all easy to use. Visit http://www.dovepress.com/ testimonials.php to read real quotes from published authors. 